\title{
The Virtual Rolling Mill - Enhancing Product Development and Commissioning
}

\author{
Maksim Klinkov ${ }^{1, a^{*}}$, Roger Feist ${ }^{1, b}$ \\ ${ }^{1}$ Achenbach Buschhütten GmbH \& Co. KG, Siegenerstr. 152, Kreuztal, 57223, Germany \\ am.klinkov@achenbach.de, ${ }^{b}$ r.feist@achenbach.de
}

Keywords: rolling mill technology, software in loop simulation, model based thickness and flatness control, virtual machine, industry 4.0.

\begin{abstract}
Achenbach supplies worldwide with first-class customized rolling mills and machinery for the manufacturing of flat-rolled products from non-ferrous metals. In order to fulfil the customer-specific production requirements, Achenbach developed a wide variety of options for specific subjects. The control of the strip quality parameters like flatness or thickness in the rolling process is a key performance index.

An increase of computation power in the field of industrial computers gave a chance to develop more complex model based control algorithms, which led to a significant improvement of the strip quality. In addition it gave an impulse for the development of a comprehensive mathematical model of the rolling mill.

This paper presents the general virtual Achenbach Rolling Mill which is a digital representation of the rolling process in general and the behavior of the major actuators in the rolling process in detail. The behavior of drives, strip-tensions, and roll-gap is simulated in a multi-variable real-time environment. In the same environment the OPTIROLLi3 ${ }^{\circledR}$ - model based controls can interact with the virtual machine. A vast number of challenges of the real rolling situation can be demonstrated by working on this 'mill simulator' and improved solutions are developed taking advantages from this platform. As the virtual machine allows all kinds of virtual testing without scrapping 'real material' a wide range of applications is possible for this virtual rolling mill. Some results from the SIL (software in loop) simulation will be presented for better clarity.
\end{abstract}

\section{Introduction}

A modern cold rolling mill shown in Fig. 1 (a) is a complex machine consisting of mechanical, electrical, hydraulic/pneumatic aggregates and measurement components (e.g. mill housing, drive systems, hydraulic cylinders, spray bars and sensors) that are fitted together in a modular way [1]. Only united work of these systems allows producing highly qualitative products in terms of thickness and flatness under tough throughput conditions (i.e. fast rolling speed, wide and heavy coils). Achenbach's mill-automation system OPTIROLLi3 ${ }^{\circledR}$ shown in Fig. 1 (b), takes care of the correct usage of all sensor information in order to produce the optimum output.

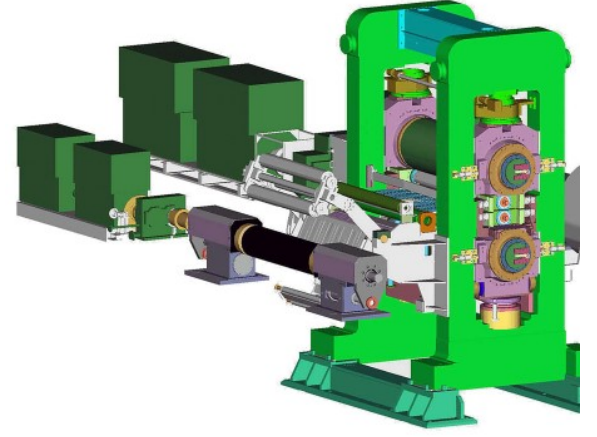

(a)

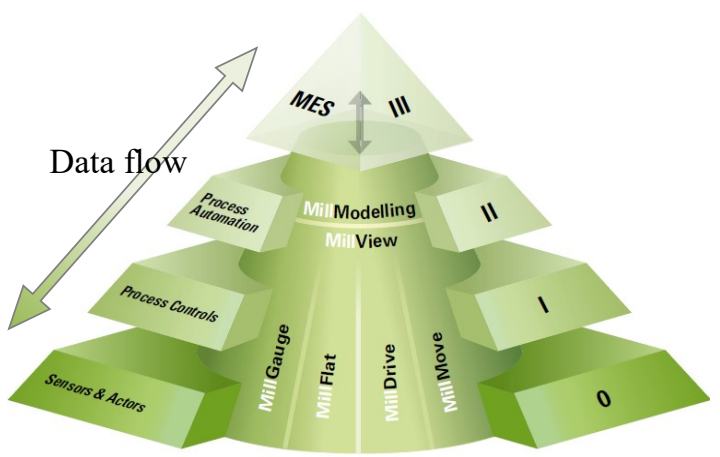

(b)

Fig. 1: (a) - Achenbach strip mill center part; (b) - OPTIROLLi3 ${ }^{\circledR}$ automation system 
Besides the classic dataflow in top-down direction with aggregation and dissolving of information, it is equipped with up-to-date interface-services as OPC-UA. Taking advantage of these services the system can provide sensor- or control-level information to virtually every client. In addition to the real-time process-data sophisticated evaluations can be carried out on the data in order to enhance product-related optimization or predictive maintenance.

Especially, "MillGauge" and "MillFlat" modules in the OPTIROLLi3 ${ }^{\circledR}$ are using advantages of the models for better control of strip thickness and flatness. A major improvement of model deployment inside of controller algorithms (called: a model based predictive controller - MBPC) is achieved by the model ability to predict the future state of the controlled process instead of simple reaction to the current error. The models that are used inside of the controller algorithms are of hybrid nature and come either from of physical laws or are obtained empirically via certain test procedure. The combination of detailed process models with the MBPC allows closing the loop and using such "virtual systems" for the controller tests during the design phase and reduces drastically commissioning time on site.

\section{Virtual Rolling Mill Architecture}

The Achenbach "virtual mill" consists of three modules: 1) models of the thickness and flatness behavior in the mill; 2) data acquisition [2], which handles the virtual coils and 3) respective controllers, see Fig. 2. The models in the $1^{\text {st }}$ module are utilized in Matlab Simulink environment while the controller methods are implemented in PLC-language [3-4]. The M-Target tool gives the possibility to deploy Simulink models into PLC and use them in real time. As a benefit of such an architecture identical controller-modules can be used inside the simulation-environment or in the real application. Additionally support-modules may be designed inside Simulink and then used within the controller-environment.

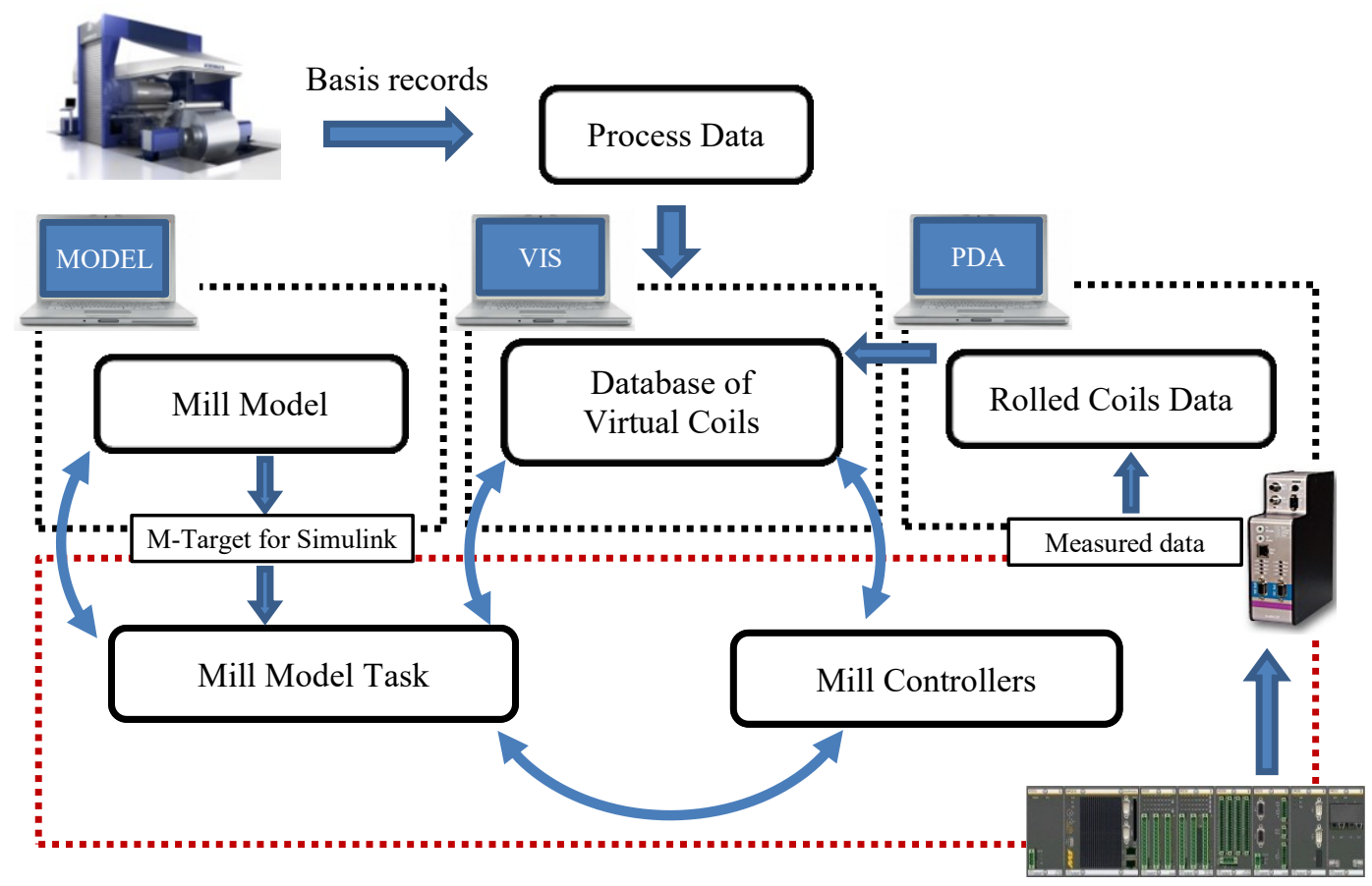

Fig. 2: The "virtual mill" architecture

Virtual Coils and Visualization. The basis coils (i.e. 5 to $8 \mathrm{~mm}$ thick strip coming from hot mill for the first rolling) that are used for the "virtual mill" are taken from the records obtained at real rolling mill and rolled down to the end product. Each pass (i.e. a single process step of thickness reduction in rolling) generates a new process data which serves for output coil creation. A newly generated output coil is used as an input coil for the next pass. The data acquisition system together with visualization software tool allows witnessing all important process values in real time. 
Mill Model. A realistic mill behavior is generated by the synergy of two models:

a) Thickness model - combines influences of the gap movement, back tension, friction, entry thickness variation as well as the mill starch and strip flexibility on the exit thickness variation [5], see Fig. 3. In addition it considers mill specific actuators i.e. electrical motors, hydraulic cylinders and sensor dynamics. An operator block in Fig. 3 allows switching between different tests scenarios.

b) Flatness model - takes into account influence of both geometrical and dynamical effects from: bending, tilting, variable crown roll and the thermic profile of the gap, on the out-coming strip flatness. The thermic profile is constructed based on heat transfer due to friction forces for specific mill states i.e. rolling speed and cooling properties of the lubrication oil.

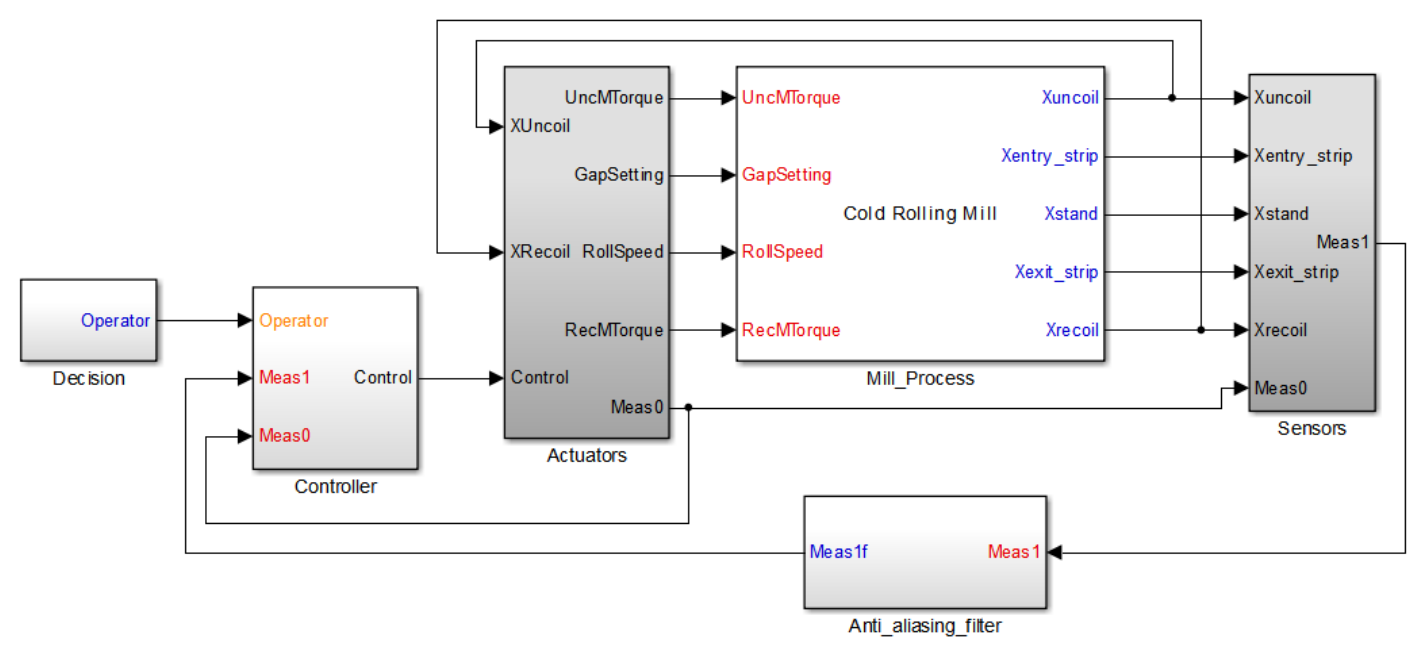

Fig. 3: Thickness module in Matlab Simulink

Model Based Controller. The control strategies applied in the rolling mill often have nested structures as shown in Fig. 4, e.g. thickness control loop with thickness as the primary control variable (CV1) together with the position of hydraulic cylinders as manipulated variables (MV) "outer loop" and the servo control loop with the hydraulic cylinders position as a secondary (CV2) - "inner loop". These loops can be driven by different control strategies e.g. PID algorithm for servo control and MBPC strategy for thickness control.

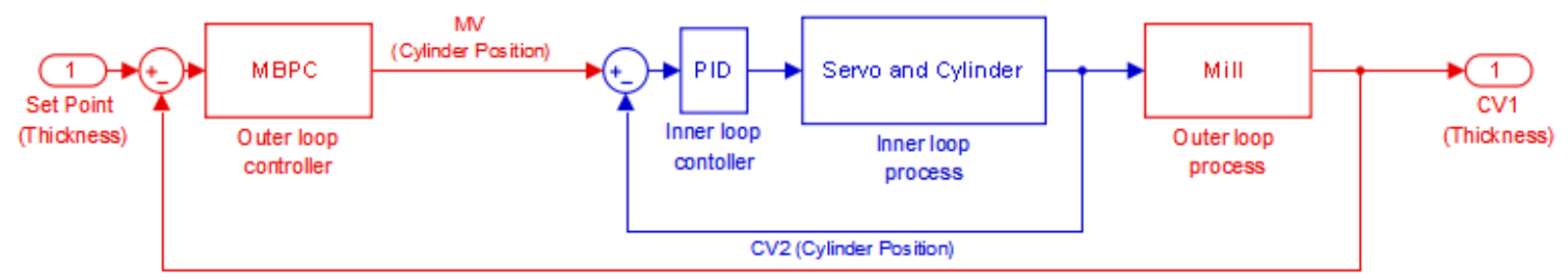

Fig. 4: Nested control architecture of the thickness control loop

In rolling industry, application of MBPC brings benefits especially due to the process variety (i.e. different material hardness, width, speed regimes), which makes tuning of PID controllers tedious and time consuming procedure. In contrast to PID the MBPC has implicit ability to adapt the controller gains based on the model and to compute the optimal MV for the current state with the consideration of the future trajectory which makes it more stable if time delays are present in the control loop (i.e. thickness or flatness always measured with delay). The biggest challenge in MBPC design is to acquire and validate a specific model of modest complexity which would be precise enough to serve for the necessary predictions. Model construction can base on physical laws, empirical trials or can be of heuristic nature. Nevertheless, once the specific model is obtained the controller algorithm is ready for tests and further use. 
For clarity, the architecture of the predictive functional control (PFC) [6], which is a subset of $\mathrm{MBPC}$, is shown in Fig. 5. A manipulated variable $\left(\mathrm{MV}^{*}\right)$ which is calculated by regulator, based on the internal model prediction and modeled disturbance (DVm) is passed through a limiter producing the applied manipulated variable (MV) output presented to the process and to the internal model of the regulator. The main objective of the PFC is to drive the controlled variable $(\mathrm{CV})$ that is combined from the pure process output and both known and unknown (DVm) disturbances (DVnm), so that it follows a desired set point (SP).

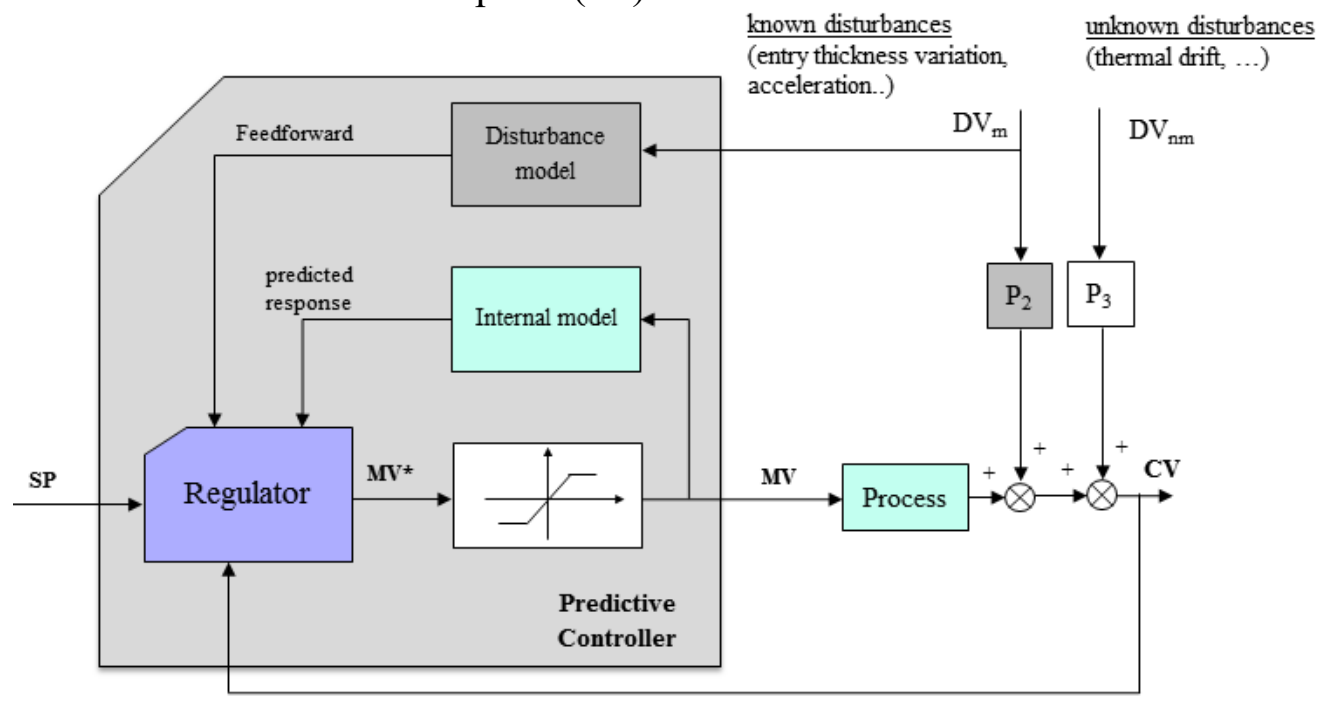

Fig. 5: Example of block diagram of predictive functional control [6] for thickness regulation

The principle of the MV computation by a simple internal model (first order transfer function) is shown in Fig. 6. The MV is computed based on the principle, that the predicted undelayed process output $\hat{y}_{p}(n+h)$ should match the reference trajectory $y_{r}(n+h)$ at coincidence point $h$ as defined in Fig. 6 (e). The reference trajectory $y_{r}(n)$ is defined as exponential error decay, as in Fig. 6 (a), which is initialized at time $n$ to overcome the delay problems. As the actual process measurements $y_{p}(n)$ are not representing the complete process-status, an advanced process output $\hat{y}_{p a v}(n)$ is used to calculate the control-error $\hat{\varepsilon}(n)$.

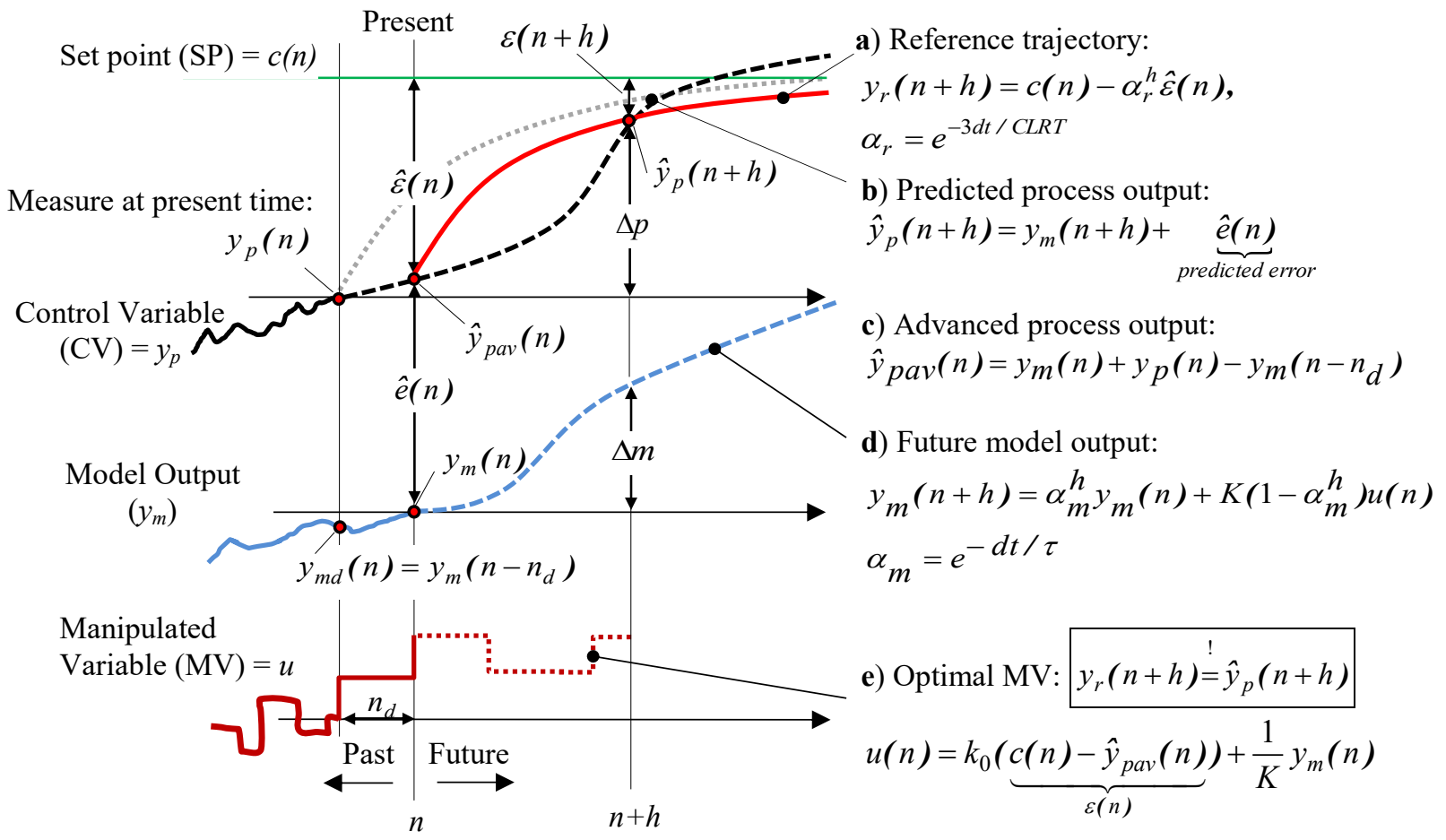

Fig. 6: Basic principle for MBPC structuring for the process with time delay 
Using the solution of the first order differential equation to predict the model response at coincidence point $h$ as in Fig. 6 (c) it is possible to find the optimal MV which will lead to a desired process output $u(n)$. The computation of the $u(n)$ is done online at each sampling time.

\section{Experimental Results on "Virtual Mill"}

The constructed "virtual mill" gives the possibility of performing the vast number SIL tests, which allows not only the controllers assessment but also assists for better understanding of the fast changing rolling mill process.

In addition to that, the simulator serves as a training tool where both the Achenbach's customers can learn to operate automation system in optimal way without scraping the material and the automation team can consider customers' needs for future improvements.

Mill Eccentricity. Every real rolling mill experiences the influence of the rolls eccentricity (i.e. irregularity in roll geometry which causes periodic variations in the roll gap) on the exit thickness and flatness. These disturbances can become critical as they are normally not compensated by the feedback loop especially if the rolling speed is high, high frequency variations are added into the outrunning material at each pass. As a result the eccentricity compensator (ECO) which is capable to diminish the effect of such disturbances is an essential part that should be present in the automation system. The effect of the roll eccentricities is related to the mill speed and diameters of the roll. This knowledge gives a possibility to incorporate the eccentricity disturbance into the "virtual mill" and use it for the controller assessment in terms of performance and stability. An example of the controller action to a dominant disturbance from the first harmonic of the backup roll (BUR1) is presented in Fig. 7. The effectiveness of the ECO in improvement of the exit thickness is obvious in time domain, Fig. 7(a) as well as in the spectrum Fig. 7 (b) and (c) for the given mill speed. With the help of simulator it is also possible to check the band width and the stability margins of the ECO by variation of the rolling conditions e.g. speed.

a) Time record of the mill response

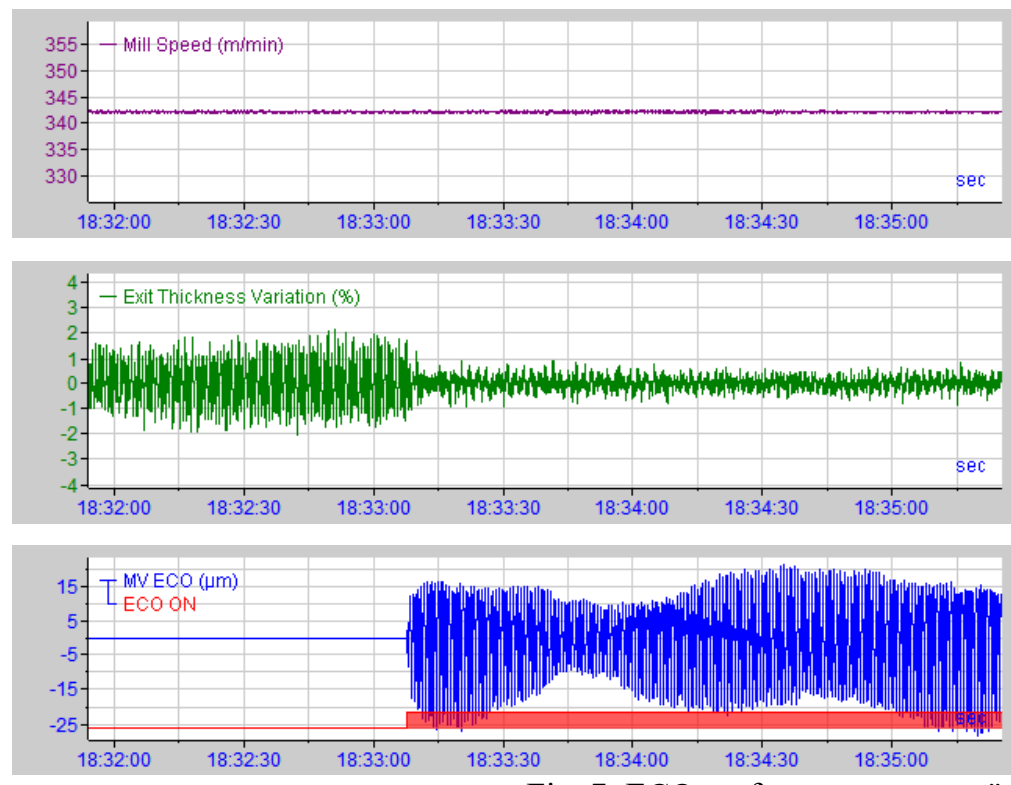

Fig. 7: ECO performance test on "virtual mill"

\section{b) FFT - ECO OFF}

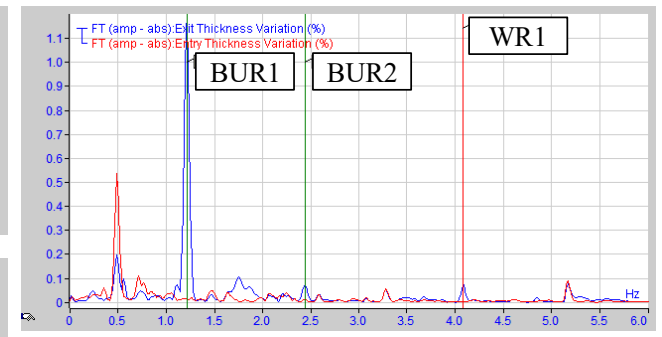

c) FFT - ECO ON

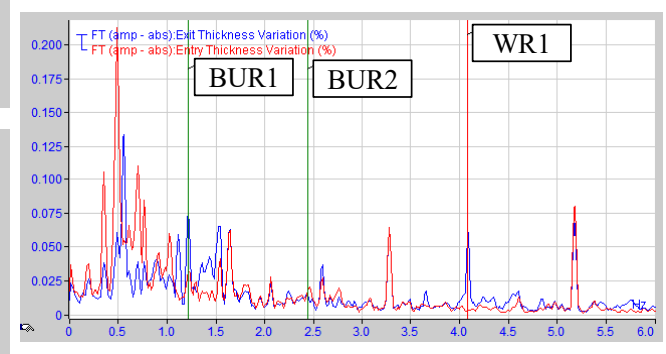

Flatness Variation Investigation. The flatness is another key quality criterion of the outrunning product. Therefore it is important that the model inside the "virtual mill" has a capability of capturing all known effects that are influencing the flatness. This, on the one hand serves for realistic mill representation and understanding of the rolling physics, on the other gave a chance to design a proper regulator algorithms. An example of the flatness variation due to the fast change in rolling force is shown in Fig. 8 (a) and (b). The change in force, Fig. 8 (c) is due to the acceleration 
of the mill which modifies the friction conditions inside the roll gap and simultaneous action of the thickness controller which pulls the exit thickness into target.

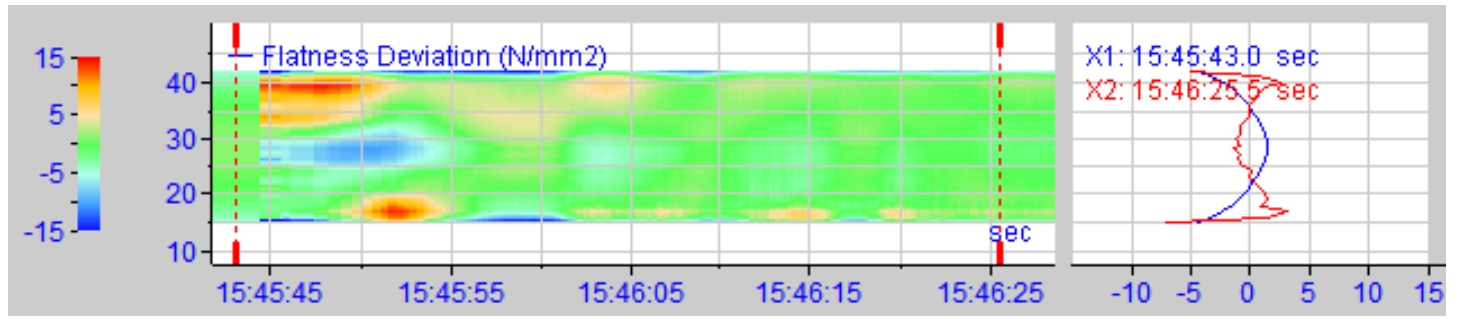

a) Flatness carpet captured by flatness roll

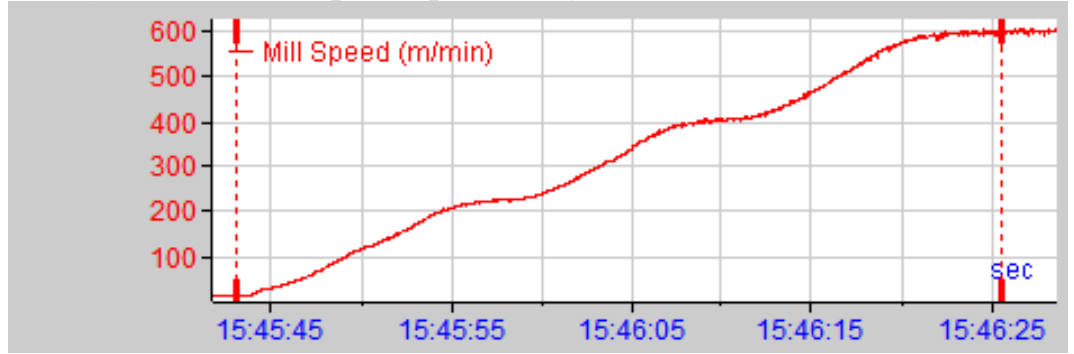

b) Mill speed

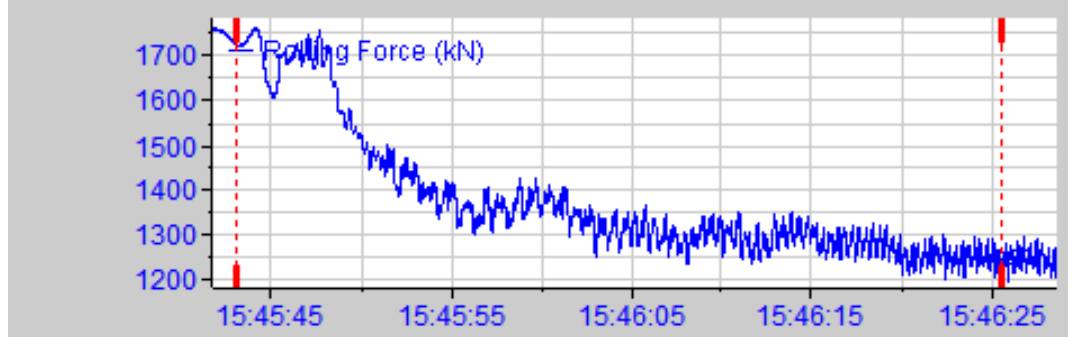

c) Rolling force

Fig. 8: Reproduction of the flatness variation in acceleration phase. Strip: thickness $0.3 \mathrm{~mm}$, width $970 \mathrm{~mm}$

\section{Summary}

Nowadays the automation technology of the modern rolling mill progressively involves modeling into process control. Comprehensive model combined into software in the loop or hardware in the loop tests allows engineers to design and validate new controller methods. The tests reduce the commissioning time and the probability of unexpected errors inside the controller algorithm.

\section{References}

[1] Axel E. Barten, Aluminium Rolling Mill Technology, Verlag Moderne Industrie, Landsberg, (2002)

[2] Information on http://www.iba-ag.com/en/products/

[3] Information on http://www.bachmann.info/produkte/

[4] DIN EN IEC 61131-3:2013 Programmable Controllers - Part 3: Programming languages

[5] P. R. Evans, Control techniques to Improve Rolling Mill Dynamics, PhD Thesis, University of Bath, (1999)

[6] H. Bouhenchir, M. Cabassud and M.V. Le Lann, Predictive functional control for the temperature control of a chemical batch reactor, Computers and Chemical Engineering Volume 30, Issues 6-7, 15 May (2006), Pages 1141-1154 